\title{
Generalized hypercementosis - A report of an incidental finding
}

\author{
Purva B. Butala ${ }^{1}$, Purv S. Patel ${ }^{2 * *}$ \\ Reader, ${ }^{1}$ Dept. of Pedodontics \& Preventive Dentistry, ${ }^{2}$ Dept. of Oral Medicine \& Radiology, Ahmedabad Dental College \& \\ Hospital, Gujarat, India \\ *Corresponding Author: \\ Email: purv57@gmail.com
}

\begin{abstract}
Hypercementosis is an idiopathic, non-neoplastic condition causing excessive deposition of normal cementum on the surface of roots of one or more teeth. It occurs due to a variety of local and systemic factors; but often goes undiagnosed because of a lack of clinical signs and symptoms and can only be diagnosed radiographically. Generalized hypercementosis is a rare occurrence with a very few cases reported in literature. It holds clinical significance with respect to extractions, endodontic treatment as well as orthodontic movement of the teeth which is much more complex for teeth with hypercementosis teeth in comparison with teeth without hypercementosis. This article is a report of such a rare case of generalized hypercementosis which was incidentally diagnosed based on radiographic examination.
\end{abstract}

Keywords: Hypercementosis, Cementum hyperplasia, Cementum.

\section{Introduction}

The term cementum is derived from the Latin word cementum or "cement," meaning the stone particles used to make mortar, ideally describing its role. The cementum covers the radicular portion of the tooth, providing attachment to the periodontal ligament fibers, for tooth articulation. ${ }^{1}$ Hypercementosis is defined as an idiopathic, non-neoplastic condition characterized by excessive buildup of normal cementum (calcified tissue) on the roots of one or more teeth. ${ }^{1-3}$ It is characterized by cementum formation beyond the physiologic limits of the tooth..$^{3-5}$

Apart from the idiopathic nature, this condition is associated with several local factors. ${ }^{1-3}$ The origin of hypercementosis is also attributed to conditions such as functional stress due to occlusion forces, continuous dental eruption, incorporation of periodontal cementicles during physiologic cementum deposition, reactionary deposition in response to periapical inflammatory processes, systemic factors such as atherosclerosis, acromegaly, deforming arthritis, hypertrophic arthritis, pituitary and thyroid diseases and Paget's disease. ${ }^{1,2,4,5}$ It is widely accepted as an agerelated phenomenon. ${ }^{1-3}$ The incidence of hypercementosis by race or population group has not yet been established. ${ }^{4-6}$

Hypercementosis may be evident on the entire root or only parts of a root. ${ }^{2}$ Whilst mandibular molars were the most commonly affected teeth by hypercementosis, furcation areas of multi-rooted teeth, anatomical grooves and concavities on roots were the most frequently affected sites. ${ }^{3,7}$ This condition may be isolated comprehending single tooth, may involve multiple teeth, or may show up as a generalized process. It has been noticed not only on erupted teeth but also on a tooth that is not erupted. ${ }^{6}$ It presents with no clinical signs or symptoms. ${ }^{1-3}$
Radiographically, hypercementosis presents with an altered root structure caused by the excessive build up of cementum around all or part of the root is evident. The affected teeth demonstrate a thickening of the root surrounded by a radiolucent periodontal ligament space with an adjacent intact lamina dura. The radiolucent shadow of the periodontal membrane and the radiopaque lamina dura are always seen on the outer border of hypercementosis, enveloping it as seen in normal cementum. ${ }^{1-3}$ Radiographically, hypercementosis does not alter the biologic width between the root surface, the periodontal ligament, and the alveolar bone. Although hypercementosis can be identified radiographically, it is not possible to estimate the amount of extra cementum present in the affected root because dentin and cementum have the same radiodensity. ${ }^{1,4-6}$

At the interface with the root surface the cementoblasts yield a slow but steady deposition of the cementum organic matrix into lamellar layers. Cementum thickening occurs by a rhythmic process: After deposition, each new layer of cementum is mineralized by the previous layer. The last layer, a nonmineralized layer, can also be called precementum. ${ }^{2,6}$ When deposition takes place prior to tooth eruption the cementum can be classified as primary. After eruption, the cementum can be defined as secondary and is formed in response to functional demands. ${ }^{1,}$ 2, 6 Microscopic studies about hypercementosis have reported thick layers of cementum characterized by deposition of symmetric, highly basophilic lines parallel to dentin surface.,5 Occasionally, atypical cementum depositions are identified in focal areas as external cementum projections. ${ }^{5}$

This is a report of a 58 year old female with an incidental radiographic finding of generalized hypercementosis associated with a history of orthodontic treatment. 


\section{Case Report}

A female Hindu patient aged 58 years reported at the outpatient department of the Oral Medicine \& Radiology Department of the institute with the chief complaint of generalized spacing in relation with all the teeth and bad breath. The patient gave a medical history of hypertension and was regularly taking medication for the same. Dental history included extractions of 14, 25, $27,35,44$ and 45 followed by fixed orthodontic treatment for a period of 3 years. She also underwent silver fillings in $26,27 \& 46$ and root canal treatment followed by crown in 36. Since the orthodontic treatment was taking too long with no significant result, the patient got the braces removed and discontinued the orthodontic treatment.

On intraoral examination, there were all teeth present except 14, 25, 27, 34, 44 and 45. [Fig. 1 \& 2] A signed informed consent was taken from the patient regarding the publishing of the photographs. There was pit and fissure caries in relation with 18,28, 38, 47 and 48. Also there were generalized plaque and calculus deposits accompanied by generalized gingival recession and periodontal pockets with severe bleeding on probing. There was generalized spacing between all the teeth. The patient was advised full mouth set of intraoral digital full mouth radiographs (FMX) in order to evaluate the root condition and for planning the orthodontic treatment.

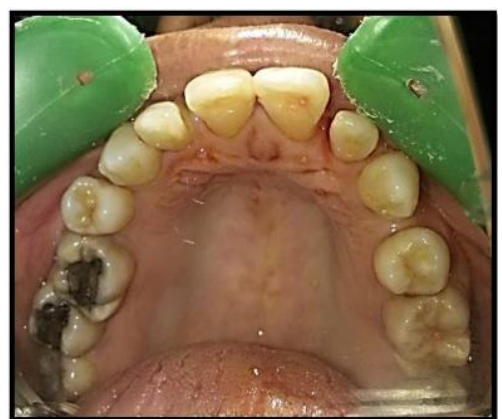

Fig. 1: Intraoral occlusal view of maxillary arch showing generalized spacing and malaligned teeth with missing 14, $25 \& 27$

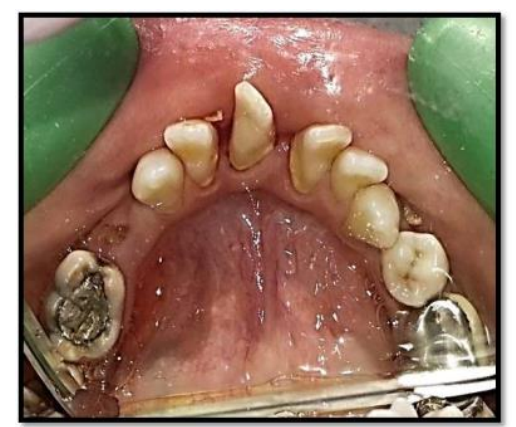

Fig. 2: Intraoral occlusal view of mandibular arch showing generalized spacing and malaligned teeth with missing $35,44 \& 45$
On radiographic examination, FMX showed that the patient had root pieces of 14 and 45. Also, there was generalized horizontal alveolar bone loss which was severe (upto midroot level) in mandibular anterior region and less severe (within the cervical third of teeth) in maxillary and mandibular posterior regions. Also, there was generalized bulbous appearance of roots of almost all the teeth except $12,11,21,22,23,27,37,32,31,41,42$ interpreted as generalized hypercementosis. [Fig. 3]

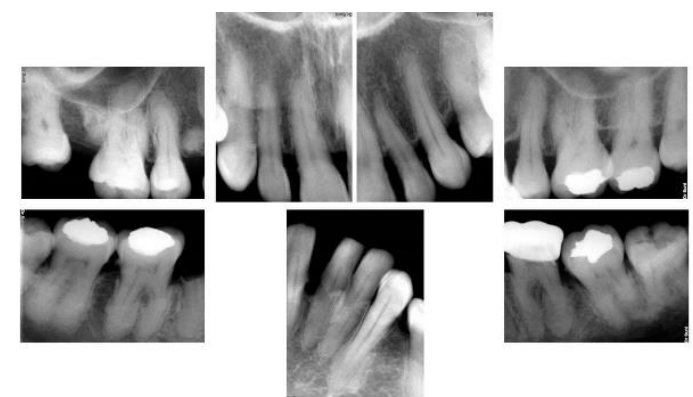

Fig. 3: Full mouth set of intraoral periapical radiographs showing generalized hypercementosis and root pieces in $14 \& 45$ region

Patient was advised full mouth scaling, deep cleaning and root planning followed by removal of root pieces of 14 and 45. After the completion of the periodontal therapy, the patient was also advised fixed orthodontic treatment as the patient was interested in getting rid of the spaces in her maxillary and mandibular anterior region for esthetic purpose. At the end of the orthodontic treatment; the space for 45, which will be maintained during the orthodontic treatment in order to prevent midline shift, will be replaced by implant and crown.

\section{Discussion}

Hypercementosis is also termed as cementum hyperplasia. ${ }^{6}$ It is an adaptive modification of the periodontium characterized by an augmented cementum thickness on the root surface beyond the level necessary to fulfill its normal function. ${ }^{2}$ This excessive amount of cementum might lead to an abnormal thickness of the apex that becomes round-shaped and/or with the root appearance altered macroscopically.,4,5 Whilst mandibular molars were the most commonly affected teeth by hypercementosis, ${ }^{7}$ the canines show very little impairment, the upper incisors are seldom affected and there are no hypercementosis cases among lower incisors. ${ }^{2}$ This is in accordance with the present case where the anterior teeth were not affected by hypercementosis.

Macroscopically, hypercementosis is classified as (a) club shaped (b) focal and (c) circular cemental hyperplasia. Generalized hypercementosis is conspicuous as increased thickness of cementum embroiling the thorough radicular surfaces of all teeth 
and is an innate feature of Paget's disease. ${ }^{6}$ Generalized hypercementosis, all be it rare, can also been observed. ${ }^{7}$ Basdra et al. (1997) reported one case of generalized hypercementosis in an 18-year-old female patient, with the need of orthodontic treatment. The patient had undergone orthodontic treatment previously. Such cases of generalized hypercementosis, in the absence of any underlying systemic factors, at such an early age have not been frequently reported in the literature. ${ }^{1}$ The present case had features of club shaped generalized hypercementosis associated with a previous history of orthodontic treatment.

The etiology of generalized hypercementosis has been put down to hereditary and systemic conditions such as Paget's disease, thyroid goiter, rheumatic fever, rheumatoid arthritis and acromegaly. In contrast to the aforementioned conditions in Paget's disease, the periodontal ligament space is also obliterated and the teeth are often ankylosed to the bone exhibiting external resorption. ${ }^{7}$ The present case was not associated with any local or systemic conditions except a history of orthodontic treatment.

Generalized hypercementosis is commonly either of the dense type or the transparent type. In the dense type, the secondary cementum has the same density as the primary cementum and dentin. In the transparent type, the demarcation between the secondary and primary cementum and dentin can be clearly visualized. ${ }^{1}$ The case under report had dense type of hypercementosis. Hypercementosis is regarded essentially in adults, and the frequency perceived to be increasing with maturing age. ${ }^{1,6}$ This is in accordance with the present case where the patient was in her sixth decade of life. Its exigency has also been divulged in younger patients, and many of these cases authenticate a positive family history that advocate a hereditary influence.

Hypercementosis must be differentiated from periapical cemental dysplasia (PCD), condensing osteitis, idiopathic osteosclerosis, cementoblastoma and rare systemic diseases like Paget's disease and metastatic carcinoma. Condensing osteitis may usually be ruled out because it occurs in the periapex of a nonvital tooth, whereas PCD does not. In addition, condensing osteitis does not have a radiolucent rim, but fibroosseous lesions of periodontal origin do. Periapical idiopathic osteosclerosis and mature PCD may be difficult to differentiate because both occur in the periapex of healthy teeth with vital pulps. However, PCD is smoothly contoured and usually round or oval, whereas periapical idiopathic osteosclerosis is usually quite irregular in shape. Also, the usual radiolucent border that can be recognized surrounding the PCD is not present in idiopathic osteosclerosis. The cementoblastoma affects the periapices of the premolars and molars almost exclusively, whereas $80 \%$ of the PCD occur in the mandibular anterior region. Most patients afflicted with Paget's disease show many radiopaque areas scattered throughout the jaws. ${ }^{8}$

Patients with hypercementosis stand in no need of any treatment. Root thickening may cause occasional botheration during the extraction of such tooth sectioning of the tooth or surgically relegate significant amounts of the surrounding bone may be necessary in such footing. Clinically, hypercementosis may also directly leverage on root canal treatment, and the clinician should appraise the necessity of adapting the usual endodontic therapy approach to assure all endodontics principles and reach treatment success. ${ }^{6}$ The case under discussion required orthodontic treatment with need for extractions of root pieces which were carried out uneventfully while taking proper precautions to avoid the need for excessive surrounding bone removal.

\section{Conclusion}

Generalized hypercementosis is a rare complication of fixed orthodontic treatment. This fact must be kept in mind while treating the patients having a previous history of orthodontic treatment. Such patients must be subjected to thorough radiographic examination as well as blood investigations to diagnose hypercementosis as well as to rule out the systemic conditions associated with hypercementosis. Furthermore, hypercementosis necessitates modifications while performing dental procedures such as extractions, endodontic treatment as well as orthodontic treatment.

\section{References}

1. Aravind W, Divya V. Irregular periapical radiopacity in mandibular premolars and molars. Case Rep Dent 2014; 1-3.

2. Satish K, Surabhi R, Sanjay J, Veerendra P, Ratnakar P. Endodontic challenges presented by a hypercementosis tooth. Int J Res Dent 2015;5(2):42-6.

3. Sudha M, Surabhi R, Soma A, Babasaheb K. A clinicopathologic review with special emphasis on hypercementosis vs. cementoblastoma. Uniq J Med Dent Sci 2014;2(4):140-1.

4. Bethania P, Tiago P, Ana C, Alberto C. A scanning electron microscopic study of hypercementosis. J Appl Oral Sci 2008;16(6):380-4.

5. Lilliana B, Bethania P, Rogerio A, Alberto C, Tiago P. Root apical third and canal morphology of teeth with hypercementosis. Dent Press Endod 2013;3(3):23-31.

6. Santosh P, Nidhi Y. Generalized hypercementosis with multiple missing teeth in a young female: A rare case report. Int J Health Allied Sci 2015;4(3):178-80.

7. Bhavya M. Hypercementosis and concrescence of maxillary second molar with third molar: a case report and review of literature. Oral Health Dent Med 2014;13(2):558-61.

8. Norman W, Paul G. Differential diagnosis of oral and maxillofacial lesions (5 ${ }^{\text {th }}$ Edition) 1997;Mosby, Missouri. 